\title{
Erratum to: An Introduction to Medical Physics
}

\author{
Muhammad Maqbool
}

\section{Erratum to:}

M. Maqbool (ed.), An Introduction to Medical Physics, Biological and Medical Physics, Biomedical Engineering, https://doi.org/10.1007/978-3-319-61540-0

The name of the editor was printed incorrectly (Muhammed Maqbool) in the previous version. The correct name of the Author is Muhammad Maqbool. 